Fructose intervention for 12 weeks does not impair glycemic control or incretin hormone responses during oral glucose or mixed meal tests in obese men

\title{
Matikainen, N.
}

2017-06

Matikainen, N, Söderlund, S, Björnson , E , Bogl , L H, Pietiläinen , K H , Hakkarainen , A , Lundbom , N, Eliasson, B , Räsänen , S , Rivellese , A, Patti , L , Prinster , A , Riccardi , G, Despres , J -P , Almeras , N , Holst, J J , Deacon , C F , Boren , J \& Taskinen , M -R 2017 , ' Fructose intervention for 12 weeks does not impair glycemic control or incretin hormone responses during oral glucose or mixed meal tests in obese men ', Nutrition, Metabolism and Cardiovascular Diseases , vol. 27 , no. 6 , pp. 534-542 . https://doi.org/10.1016/j.numecd.2017.03.0

http://hdl.handle.net/10138/237043

https://doi.org/10.1016/j.numecd.2017.03.003

unspecified

publishedVersion

Downloaded from Helda, University of Helsinki institutional repository.

This is an electronic reprint of the original article.

This reprint may differ from the original in pagination and typographic detail.

Please cite the original version. 


\title{
Fructose intervention for 12 weeks does not impair glycemic control or incretin hormone responses during oral glucose or mixed meal tests in obese men
}

\author{
N. Matikainen a,b,*,1, S. Söderlund ${ }^{\mathrm{a}, 1}$, E. Björnson ${ }^{\mathrm{c}}$, L.H. Bogl ${ }^{\mathrm{a}, \mathrm{d}, \mathrm{e}}$, K.H. Pietiläinen ${ }^{\mathrm{a}, \mathrm{b}}$,
} A. Hakkarainen ${ }^{\text {f }}$, N. Lundbom ${ }^{\text {f }}$, B. Eliasson ${ }^{\text {c}}$, S.M. Räsänen ${ }^{\text {a }}$, A. Rivellese ${ }^{\mathrm{g}}$, L. Patti $^{\mathrm{g}}$, A. Prinster ${ }^{\mathrm{h}}$, G. Riccardi ${ }^{\mathrm{g}}$, J.-P. Després ${ }^{\mathrm{i}}$, N. Alméras ${ }^{\mathrm{i}}$, J.J. Holst ${ }^{\mathrm{j}}$, C.F. Deacon ${ }^{\mathrm{j}}$, J. Borén ${ }^{c, 2}$, M.-R. Taskinen ${ }^{\mathrm{a}, 2}$

\footnotetext{
${ }^{a}$ Research Programs Unit, Diabetes and Obesity, University of Helsinki, Helsinki University Hospital, Helsinki, Finland

${ }^{b}$ Endocrinology, Abdominal Center, Helsinki University Central Hospital, University of Helsinki, Helsinki, Finland

${ }^{c}$ Department of Molecular and Clinical Medicine/Wallenberg Laboratory, University of Gothenburg, Sahlgrenska University Hospital, Gothenburg, Sweden

${ }^{d}$ Institute for Molecular Medicine FIMM, Helsinki, Finland

${ }^{e}$ Department of Public Health, University of Helsinki, Helsinki, Finland

${ }^{f}$ Radiology, HUS Medical Imaging Center, Helsinki University Hospital, University of Helsinki, Finland

${ }^{g}$ Department of Clinical Medicine and Surgery, Federico II University, Naples, Italy

${ }^{h}$ Biostructure and Bioimaging Institute, National Research Council, Naples, Italy

${ }^{i}$ Institut Universitaire de Cardiologie et de Pneumologie de Québec, Québec City, Québec, Canada

${ }^{j}$ NNF Centre for Basic Metabolic Research, Department of Biomedical Sciences, Faculty of Health and Medical Sciences, University of Copenhagen, Copenhagen, Denmark
}

Received 9 January 2017; received in revised form 28 February 2017; accepted 9 March 2017

Available online 18 March 2017

KEYWORDS
Glucagon-like
peptide 1;
Glucose-dependent
insulinotropic
polypeptide;
Fructose
intervention;
Incretin response;
Liver fat;
Metabolic syndrome;
Mixed meal test;
Oral glucose
tolerance test

\begin{abstract}
Background and aims: Incretin hormones glucagon-like peptide (GLP)-1 and glucosedependent insulinotropic polypeptide (GIP) are affected early on in the pathogenesis of metabolic syndrome and type 2 diabetes. Epidemiologic studies consistently link high fructose consumption to insulin resistance but whether fructose consumption impairs the incretin response remains unknown.

Methods and results: As many as 66 obese (BMI $26-40 \mathrm{~kg} / \mathrm{m}^{2}$ ) male subjects consumed fructosesweetened beverages containing $75 \mathrm{~g}$ fructose/day for 12 weeks while continuing their usual lifestyle. Glucose, insulin, GLP-1 and GIP were measured during oral glucose tolerance test (OGTT) and triglycerides (TG), GLP-1, GIP and PYY during a mixed meal test before and after fructose intervention. Fructose intervention did not worsen glucose and insulin responses during OGTT, and GLP-1 and GIP responses during OGTT and fat-rich meal were unchanged. Postprandial TG response increased significantly, $\mathrm{p}=0.004$, and we observed small but significant increases in weight and liver fat content, but not in visceral or subcutaneous fat depots. However, even the subgroups who gained weight or liver fat during fructose intervention did not worsen their glucose, insulin, GLP-1 or PYY responses. A minor increase in GIP response during OGTT occurred in subjects who gained liver fat $(\mathrm{p}=0.049)$.

Conclusion: In obese males with features of metabolic syndrome, 12 weeks fructose intervention $75 \mathrm{~g} /$ day did not change glucose, insulin, GLP-1 or GIP responses during OGTT or GLP-1, GIP or
\end{abstract}

Abbreviations: AUIC, area under incremental curve; AUC, area under curve; GIP, glucose-dependent insulinotropic polypeptide; GLP-1, glucagon-like peptide-1; HDL, high density lipoproteins; LDL, low density lipoproteins; OGTT, oral glucose tolerance test; PYY, peptide YY; TG, triglyceride.

* Corresponding author. Research Programs Unit, Diabetes and Obesity, University of Helsinki, Helsinki University Hospital, Helsinki, Finland.

E-mail address: niina.matikainen@hus.fi (N. Matikainen).

${ }^{1}$ Shared first authorship.

${ }^{2}$ Shared last authorship. 
PYY responses during a mixed meal. Therefore, fructose intake, even accompanied with mild weight gain, increases in liver fat and worsening of postprandial TG profile, does not impair glucose tolerance or gut incretin response to oral glucose or mixed meal challenge.

(c) 2017 The Italian Society of Diabetology, the Italian Society for the Study of Atherosclerosis, the Italian Society of Human Nutrition, and the Department of Clinical Medicine and Surgery, Federico II University. Published by Elsevier B.V. All rights reserved.

\section{Introduction}

An increase in the prevalence in obesity and diabetes closely parallels the dramatic rise in sugar, especially fructose, intake due to consumption of sugar-sweetened beverages (SSBs) that accounts for nearly one-half of all added sugar intake in the U.S. [1]. Nowadays, fructose is a major sweetener in Western diets [2]. In the U.S., 50\% of population consumes SSBs daily, and 25\% obtains at least $200 \mathrm{kcal}$ from these beverages [1].

The relationship between SSB and cardiometabolic diseases seen in epidemiological studies reflects potential combined effects of fructose and glucose or unique effects of fructose alone. The current data suggest a causative relationship between the intake of sweeteners, particularly fructose, and the increased prevalence of nonalcoholic fatty liver disease and dyslipidemia [1,3,4], especially postprandial plasma triglycerides (TG) [5].

Whether fructose consumption directly causes insulin resistance is much debated, and despite epidemiological evidence suggesting a link, data from intervention studies are inconsistent [4,6-13]. However, most studies included relatively small numbers of participants, a short intervention period or used only fasting insulin as a marker for insulin sensitivity. A 3-week consumption of fructose in 9 normal-weight young males impaired insulin sensitivity, measured as suppression of hepatic glucose production during a clamp, but did not change fasting insulin or glucose levels [13]. Insulin sensitivity, assessed from fasting insulin levels and deuterated glucose disposal, decreased after a 10 -week fructose consumption in overweight/obese humans [12]. Studies assessing the effect of hypercaloric fructose consumption with respect to both liver fat and insulin resistance have mostly reported no change in insulin sensitivity, with varying effects on liver fat [6-10]. One study with 6-7 days intervention reported worsened insulin resistance together with increased liver fat [11].

Gut incretins, mainly glucagon-like peptide (GLP)-1 and glucose-dependent insulinotropic polypeptide (GIP), mediate the insulinotropic response to intraluminal nutrients [14]. Defects in either secretion or insulinotropic actions of GLP-1 and GIP are early signs of acquired insulin resistance characterizing the metabolic syndrome $[5,16]$. Data on whether a single dose of fructose stimulates GLP-1 secretion in humans are inconsistent [17-19], whereas GIP secretion remains unaffected [17-19]. However, no data exists on the effect of longer fructose intervention on GLP1 and GIP responses to nutritional stimuli.
Here, we test the hypothesis whether worsening of glycemic control induced by fructose consumption (75 g/ day for 12 weeks served as a lemonade together with habitual ad libitum diet) may involve a gut incretindependent mechanism. Therefore, we studied the responses of GLP-1 and GIP in the fasting state and following a standard oral glucose tolerance test (OGTT) and the more physiological mixed meal test before and after fructose intervention in overweight male subjects who have high risk for future diabetes.

\section{Methods}

\section{Study cohort}

We recruited 82 obese healthy men to the Fructose intervention study (Clinical Trials NCT01445730) at four centers including in Quebec, Canada; Helsinki, Finland; Naples, Italy; and Gothenburg, Sweden. Two subjects discontinued the intervention study: one due to skin rash which was considered as possible allergic reaction to flavoring. The rash disappeared after the discontinuation. The other subject discontinued the study in response to his dentist's advice. In addition, the data from magnetic resonance examinations at baseline or after the fructose intervention were not sufficient from 9 subjects to allow the analyses of different fat depots and the incretin measurements after OGTT or mixed meal were lacking from 5 subjects for technical reasons. Thus, 66 subjects completed the full study protocol except for one subject, who lacked insulin measurements during OGTT. Subjects were recruited via newspaper advertisements. Inclusion criteria were the following; men with large waist circumference $(>96 \mathrm{~cm})$, body mass index (BMI) between 27 and $40 \mathrm{~kg} / \mathrm{m} \mathrm{[2],} \mathrm{and}$ stable weight $( \pm 3 \mathrm{~kg})$ for at least the last 3 months, LDL$\mathrm{C}<4.5 \mathrm{mmol} / \mathrm{l}$ and TG $<5.5 \mathrm{mmol} / \mathrm{l}$. Exclusion criteria were: age $<20$ years or $>65$ years, BMI or lipid levels outside the inclusion ranges, smoking, alcohol consumption over 2 doses/day (i.e., $20 \mathrm{~g}$ pure alcohol), type 2 diabetes, cardiovascular disease, hormonal therapy, hepatic and renal diseases, gastroenterological, thyroid or hematological abnormalities, and any chronic disease requiring medication except for controlled hypertension. None of the subjects used any medication or hormones known to influence glucose or lipoprotein metabolism. The study design was approved by the local Ethics committees and each subject gave written informed consent before participation in the study. All the samples were collected in accordance with the Declaration of Helsinki. 


\section{Fructose intervention}

Each subject underwent a 12-week fructose intervention period, during which they consumed $75 \mathrm{~g}$ of fructose daily. Fructose was administered as three daily $330 \mathrm{ml}$ bottles equaling $990 \mathrm{ml}$ and $303 \mathrm{kcal}$ per day. The carbonated beverages were prepared as $7.6 \%(\mathrm{w} / \mathrm{w})$ solutions and flavored with lemon aroma (specially produced for this study by Nokian Panimo Oy, Finland). Subjects were instructed to consume the beverages together with the three main meals while continuing their habitual ad libitum diet and physical activity during the intervention. The fructose-sweetened beverage was well tolerated. Each subject kept a 3-day food record (2 work days and 1 day off) before the fructose intervention period and again within 2 weeks before completing the intervention period. A qualified nutritionist gave detailed verbal and written instructions for filling in the food records and contacted the participants every $1-2$ weeks to assure compliance with fructose intervention and lifestyle.

\section{Study design}

The subjects participated in 3 separate study visits within 2 weeks before the fructose intervention period: 1 ) oral glucose tolerance test (OGTT), 2) mixed meal test, and 3) magnetic resonance imaging. These visits were repeated within 2 weeks before completing the 12-week fructose intervention period. The OGTT $(75 \mathrm{~g})$ was performed after an overnight fast. Subjects consumed $75 \mathrm{~g}$ of glucose and blood sampling was done before and at 5, 10 and $30 \mathrm{~min}$, and 1, 1.5, 2, 3 and $4 \mathrm{~h}$. The standardized mixed meal test was performed at each center after an overnight fast. The subjects received a mixed meal test consisting of bread, butter, cheese, ham, boiled eggs, fresh red pepper, low fat (1\%) milk, orange juice and tea or coffee (63 g carbohydrates, $56 \mathrm{~g}$ fat and $40 \mathrm{~g}$ protein). The fat-rich meal contained $927 \mathrm{kcal}$. Blood samples were drawn before and at $0.5,1,2,3,4,6$, and $8 \mathrm{~h}$ after the meal. During the test, only water was allowed ad libitum and the subjects remained physically inactive. The participants abstained from alcohol and physical exercise for two days before each examination.

\section{Determination of liver, subcutaneous and visceral fat depots}

Proton magnetic resonance spectroscopy was performed with a $1.5 \mathrm{~T}$ whole-body device to determine liver fat content [20]. A standardized protocol was used at all centers, and all analyses of the imaging results were performed by one person (AH). Subjects were advised to fast for $4 \mathrm{~h}$ before imaging.

\section{Biochemical analyses}

TG and cholesterol concentrations in total plasma were analyzed by automated enzymatic methods using the
Konelab 60i analyzer (Thermo Fisher Scientific Inc., Espoo, Finland). Concentrations of glucose (hexokinase method, Roche Diagnostic Gluco-quant, Mannheim, Germany). Insulin (electrochemiluminescence with Roche sandwich immunoassay on a Cobas autoanalyzer) was measured during OGTT.

GLP-1 and GIP plasma concentrations were measured after ethanol extraction (70\% vol/vol, final concentration) using C-terminally directed assays, which detect both the intact peptide and the primary ( $\mathrm{N}$-terminally truncated) metabolite; such assays therefore can be designated as "total", and the results can be interpreted to reflect the rate of secretion [21]. For GIP [22], we used antiserum code \# 80867, which reacts fully with GIP 3-42, but not with GIP 8000 , whose chemical nature and relationship to GIP secretion is uncertain. Plasma concentrations of GLP-1 were measured [23] using antiserum code no. 89390, which is specific for the amidated C-terminus of GLP-1. PYY concentrations were measured during mixed meal (Fasting and $4 \mathrm{~h}$ ) with Elisa method (Merck, Darmstadt, Germany).

\section{Statistical analyses and calculations}

Statistical analyses were performed using IBM SPSS Statistics (Version 22, NY, USA). Data are presented as mean \pm SEM or standard deviation and range. HOMA-IR indices were calculated as described previously [24]. A web-based calculator was used to calculate Matsuda Index and Insulinogenic Index during OGTT (http://mmatsuda. diabetes-smc.jp/MIndexsi.html). Comparisons between before and after fructose intervention were evaluated with paired nonparametric tests (the Wilcoxon Matched-Pair Signed-Rank test to continuous data when comparing two specified variables and Friedman's 2-Way ANOVA by Ranks to continuous data when comparing more than 2 specified variables). In case of significant differences in 2Way ANOVA, post hoc analysis was done with Scheffe's method. The area under curve (AUC) and incremental AUC (i.e., the AUC above the fasting concentration; AUIC) for postprandial variables were calculated according to the trapezoid rule. Correlations were calculated using Spearman's rank test. A $p$ value $<0.05$ was considered statistically significant.

\section{Results}

\section{Baseline characteristics}

The study subjects were on average 48 years old and had a BMI of $30.6 \mathrm{~kg} / \mathrm{m}^{2}$. Their characteristics are summarized in Table 1 . Before the OGTT or mixed meal, fasting concentrations of glucose, insulin, GLP-1 and GIP were essentially similar. There were no correlations between fasting concentrations of GLP-1 or GIP with BMI, liver fat, subcutaneous or visceral fat nor with concentrations of fasting glucose or insulin. Only fasting GLP-1 correlated with HOMA-IR $(r=0.245, p=0.049)$. 
Table 1 Subject characteristics before and after the fructose intervention period. Data are mean \pm standard error of mean and range.

\begin{tabular}{|c|c|c|c|c|c|c|c|}
\hline & \multicolumn{3}{|l|}{ Before } & \multicolumn{3}{|l|}{ After } & \multirow[t]{2}{*}{ p-value } \\
\hline & Mean & SEM & Range & Mean & SEM & Range & \\
\hline Age, y & 49.0 & 1.2 & $21-65$ & & & & \\
\hline BMI, $\mathrm{kg} / \mathrm{m}^{2}$ & 30.6 & 0.3 & $26.5-38.2$ & & & & \\
\hline Weight, kg & 99.2 & 1.4 & $65.2-135$ & 100.0 & 1.5 & $66.8-136$ & 0.002 \\
\hline fP-Glucose, mmol/l & 5.45 & 0.06 & $4.50-6.50$ & 5.54 & 0.07 & $4.30-7.00$ & 0.090 \\
\hline fP-Insulin, pmol/l & 98.3 & 7.0 & $19.0-293.0$ & 98.2 & 7.4 & $13.0-358.0$ & 0.987 \\
\hline HOMA-IR & 4.0 & 0.3 & $0.9-13.7$ & 4.1 & 0.3 & $0.5-15.1$ & 0.710 \\
\hline HOMA-S & 143.2 & 9.2 & $20.4-408.4$ & 135.7 & 9.4 & $21.3-453.6$ & 0.216 \\
\hline Matsuda Index & 3.43 & 0.23 & $0.85-9.42$ & 3.61 & 0.30 & $0.70-13.55$ & 0.961 \\
\hline Insulinogenic Index & 1.25 & 0.12 & -1.28 to 4.72 & 1.25 & 0.12 & -0.02 to 7.09 & 0.645 \\
\hline fP-GLP-1 (OGTT), pmol/l & 13.8 & 0.7 & $4.0-31.0$ & 13.7 & 0.6 & $6.0-29.0$ & 0.767 \\
\hline fP-GLP-1 (mixed meal), pmol/l & 13.5 & 0.7 & $3.0-37.0$ & 13.6 & 0.7 & $4.0-31.0$ & 0.051 \\
\hline fP-GIP (OGTT), pmol/l & 13.9 & 2.3 & $3.0-154.0$ & 12.3 & 0.6 & $3.0-27.0$ & 0.322 \\
\hline fP-GIP (mixed meal), pmol/l & 11.1 & 0.6 & $3.0-29.0$ & 12.2 & 0.7 & $2.0-27.0$ & 0.070 \\
\hline $\mathrm{fS}-\mathrm{TG}, \mathrm{mmol} / \mathrm{l}$ & 1.56 & 0.11 & $0.34-4.52$ & 1.66 & 0.11 & $0.46-4.25$ & 0.032 \\
\hline Liver fat, \% & 6.9 & 0.8 & $0.3-24.8$ & 7.5 & 0.8 & $0.6-27.7$ & 0.021 \\
\hline Visceral fat, $\mathrm{cm}^{3}$ & 2487 & 117 & $538-5434$ & 2513 & 111 & $612-5198$ & 0.621 \\
\hline Subcutaneous fat, $\mathrm{cm}^{3}$ & 4223 & 169 & $1820-8491$ & 4243 & 176 & $1832-8389$ & 0.131 \\
\hline fP-PYY, pg/ml & 107.7 & 7.8 & $28.3-369.2$ & 100.1 & 6.4 & $32.8-247.3$ & 0.220 \\
\hline P-PYY 4 h, pg/ml & 164.6 & 9.4 & $31.1-496.9$ & 165.5 & 8.5 & $54.1-384.7$ & 0.856 \\
\hline
\end{tabular}

Fructose intervention does not modify indexes of insulin sensitivity or secretion or responses of glucose, insulin or incretins during OGTT

During the standard OGTT, the responses (both AUC and AUIC) of glucose, insulin, GLP-1 and GIP were essentially similar before and after fructose intervention (Fig. 1). In accordance, fasting HOMA-IR Matsuda Index, Insulinogenic
Index or Disposition Index did not change significantly during fructose intervention (Table 1).

\section{Fasting or postprandial PYY concentrations are not affected by fructose intervention}

PYY concentrations rose similarly by 65 and $60 \%$ ( $\mathrm{p}<0.001$ ) from fasting to $4 \mathrm{~h}$ during mixed meal before
A
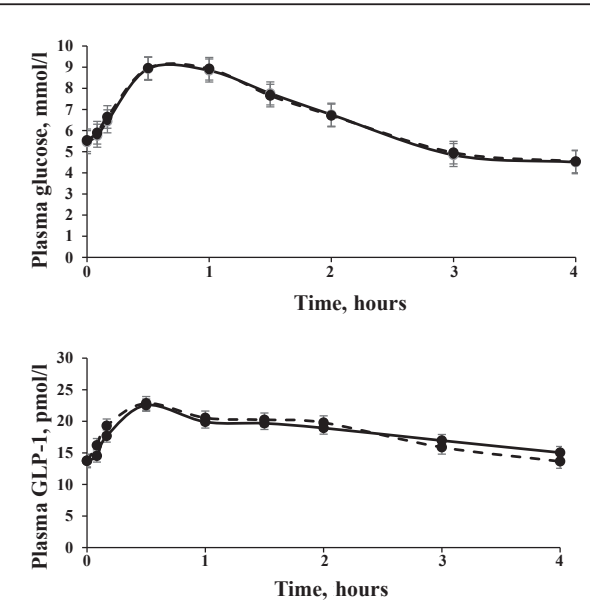
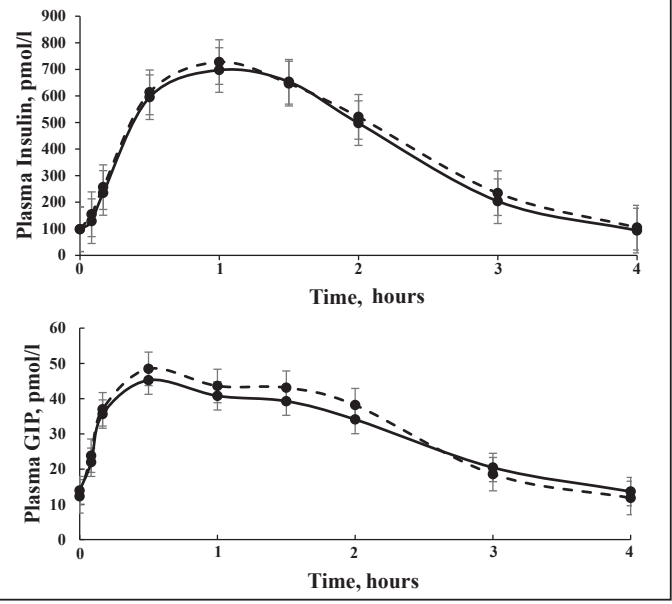

Time, hours

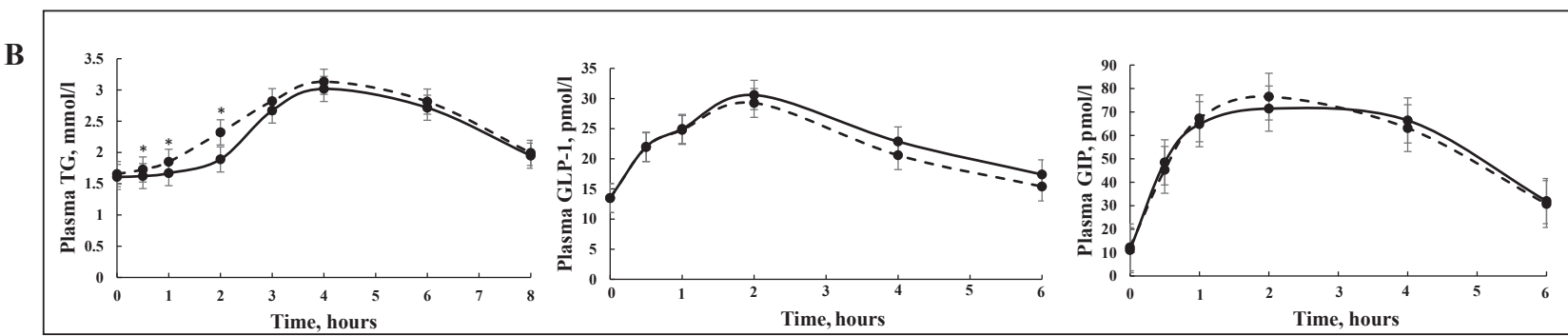

Figure 1 Panel A. Responses of glucose, insulin, GLP-1 and GIP after OGTT before (solid line) and after fructose intervention (dashed line). Panel B. Responses of triglycerides, GLP-1 and GIP after mixed meal test before (solid line) and after fructose intervention (dashed line). Data show mean \pm SEM. 
and after fructose intervention, respectively. The 12 weeks fructose intervention did not have an impact on PYY levels. The data are presented in Table 1.

\section{Fructose intervention increases postprandial responses of triglycerides but not that of incretins during mixed meal}

Fructose intervention increased fasting TG concentration slightly but significantly (Table 1). The postprandial TG responses differed before and after fructose intervention, with the response of plasma TG being higher after fructose intervention (Table 2). The difference was due to higher plasma TG values at $0,0.5,1$ and $2 \mathrm{~h}$ after the fructose intervention (Table 1, Fig. 1). The AUC or AUIC responses of GLP-1 and GIP during the mixed meal did not differ before or after fructose intervention, but peak concentrations for both GLP-1 and GIP occurred later during the mixed meal (at $2 \mathrm{~h}$ ) as compared to peak at $0.5 \mathrm{~h}$ during the OGTT.

\section{Impact of fructose intervention on diet composition, body weight, liver fat and fat depots}

Based on 3-day food records, the subjects adjusted their diets and the increase in energy intake during the fructose intervention was small (only $58 \mathrm{kcal}$ ) and did not reach statistical significance. Fructose intake at baseline was $14.5 \pm 1.0 \mathrm{~g} /$ day and increased to $85.7 \pm 0.8 \mathrm{~g} /$ day during the fructose intervention. The proportion of energy from total carbohydrates was higher, but that of sucrose, protein, total fat, and saturated and unsaturated fatty acids was lower during the fructose intervention period. The detailed intake of energy and macronutrients are shown in Supplementary Table 2.

Table 2 Glucose, insulin and incretin responses after OGTT and incretin and TG responses after a mixed meal test. Areas under curve (AUC) and areas under incremental curve (AUIC) are given before and after 12 weeks fructose intervention. Data are mean \pm standard error of mean. The given p-value shows differences between before and after fructose measurement (Wilcoxon signed rank test).

\begin{tabular}{|c|c|c|c|c|c|}
\hline & \multicolumn{2}{|l|}{ Before } & \multicolumn{2}{|l|}{ After } & \multirow[b]{2}{*}{$\mathrm{p}$} \\
\hline & Mean & SEM & Mean & SEM & \\
\hline \multicolumn{6}{|l|}{ OGTT } \\
\hline Glucose AUC, mmol/l h & 1574 & 33 & 1583 & 34 & 0.740 \\
\hline Glucose AUIC, mmol/l h & 266 & 28 & 254 & 26 & 0.496 \\
\hline Insulin AUC, $\mathrm{pmol} / \mathrm{l} \mathrm{h}$ & 96,640 & 7896 & 101,409 & 8839 & 0.722 \\
\hline Insulin AUIC, $\mathrm{pmol} / \mathrm{l} \mathrm{h}$ & 73,050 & 6987 & 77,849 & 7929 & 0.801 \\
\hline GLP-1 AUC, pmol/l h & 4401 & 182 & 4405 & 139 & 0.535 \\
\hline GLP-1 AUIC, pmol/l h & 1099 & 115 & 1110 & 113 & 0.587 \\
\hline GIP AUC, pmol/l h & 7293 & 357 & 7611 & 372 & 0.067 \\
\hline $\begin{array}{l}\text { GIP AUIC, pmol/l h } \\
\text { Mixed meal }\end{array}$ & 3955 & 455 & 4662 & 291 & 0.126 \\
\hline TG AUC, mmol/l h & 1128 & 73 & 1238 & 74 & 0.004 \\
\hline TG AUIC, mmol/l h & 379 & 30 & 441 & 35 & 0.025 \\
\hline GLP-1 AUC, pmol/l h & 8525 & 358 & 8321 & 326 & 0.522 \\
\hline GLP-1 AUIC, pmol/1 h & 3671 & 254 & 3418 & 305 & 0.305 \\
\hline GIP AUC, pmol/1 h & 20,848 & 1023 & 21,522 & 1144 & 0.387 \\
\hline GIP AUIC, pmol/l h & 16,860 & 973 & 17,125 & 1065 & 0.814 \\
\hline
\end{tabular}

In general, 12 weeks fructose intervention increased the weight slightly, but statistically significantly from 99.2 to $100.0 \mathrm{~kg}(\mathrm{p}<0.002)$. The relative increase of liver fat was $9.2 \%$ (absolute increase from $6.9 \%$ to $7.5 \%, p=0.021$ ) during fructose intervention. However, fructose intervention did not change the subcutaneous $(\mathrm{p}=0.131)$ or visceral $(\mathrm{p}=0.526)$ fat depots (Table 1$)$.

\section{Impact of weight and liver fat changes on incretin response during fructose intervention}

Because weight and liver fat changes are important determinants of glycemic control, we divided the study population to three subgroups according to 1 ) weight change and 2) liver fat change. Weight groups were: weight stable group $(\mathrm{n}=30)$ with $0-1 \mathrm{~kg}$ change, weight gain group ( $\mathrm{n}=27$ ) with $\geq 1 \mathrm{~kg}$ increase and weight loss group ( $\mathrm{n}=9$ ) with $\geq 1 \mathrm{~kg}$ decrease in body weight during the fructose intervention. Liver fat groups were: Stable liver fat group with no or minimal change from 3.7\% to $4.1 \%(\mathrm{n}=17)$, increased liver fat group with liver fat gain from $8.7 \%$ to $11.3 \%(\mathrm{n}=27)$ and reduced liver fat group with decrease in liver fat from $7.1 \%$ to $5.4 \%(n=22)$.

In the whole study group, fructose intervention did not alter any measurement of glucose metabolism but we wanted to exclude the effect of weight on OGTT responses. We therefore analyzed the weight stable, weight gain and weight loss groups separately but the results remained unchanged with no statistical difference between glucose, insulin or GIP AUC or AIUC before vs. after fructose intervention in any of the weight change groups (see Fig. 2 for AUC data). Likewise, the incretin responses as well as PYY responses during mixed meal test were similar before and after fructose in each weight group but the TG AUC was significantly higher in the weight gain group (data not shown).

Because the liver fat content is closely linked to insulin sensitivity and a predictor of diabetes, we wanted to exclude the possibility that subjects with liver fat gain respond to fructose intervention differently compared to subjects who do not increase or even decreased liver fat during fructose intervention. The metabolic characteristic including liver fat changes are given in Supplementary Table 1 . These three groups did not show any statistically difference in weight, fasting glucose, visceral fat, subcutaneous fat or fasting TG at baseline or after fructose intervention. However, in the liver fat increase group, fasting insulin and HOMA-IR were higher than in the other two groups and these changes persisted during fructose intervention. Despite the diverse liver fat responses and $5.9 \%$ difference in liver fat after the fructose intervention between liver fat decrease and liver fat increase groups, there were no differences in glucose, insulin or GLP-1 responses during OGTT, although the GIP AUC increased slightly in the increased liver fat group ( $p=0.049$, Fig. 3). During mixed meal test there were no changes in $4 \mathrm{~h}$ PYY concentrations or GLP-1 and GIP AUCs during fructose intervention, but as expected the TG AUC increased, which was statistically significant in the liver fat increase group $(\mathrm{p}=0.015)$. 


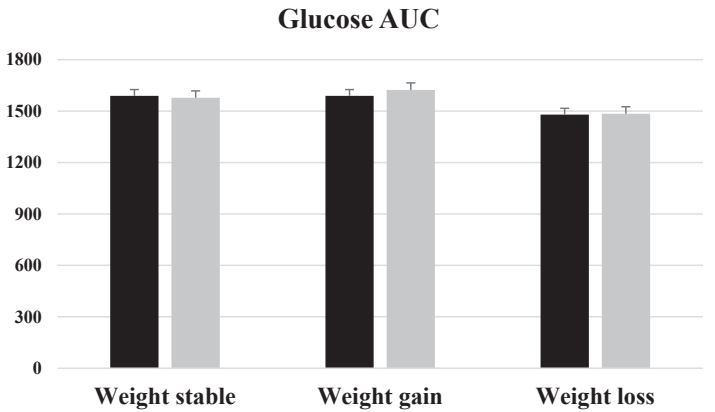

GLP-1 AUC

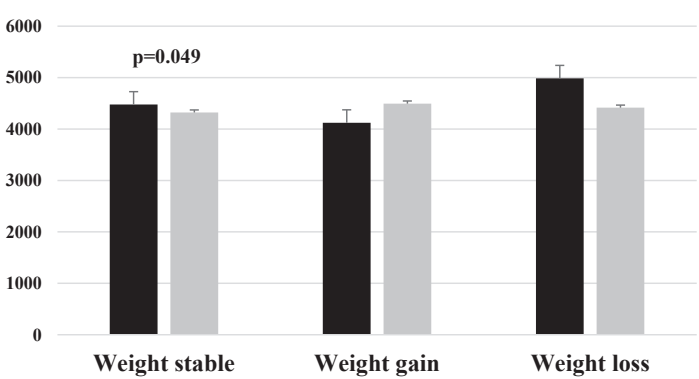

Insulin AUC

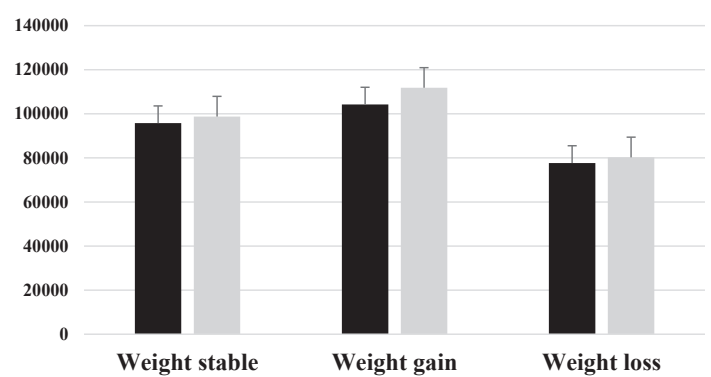

GIP AUC

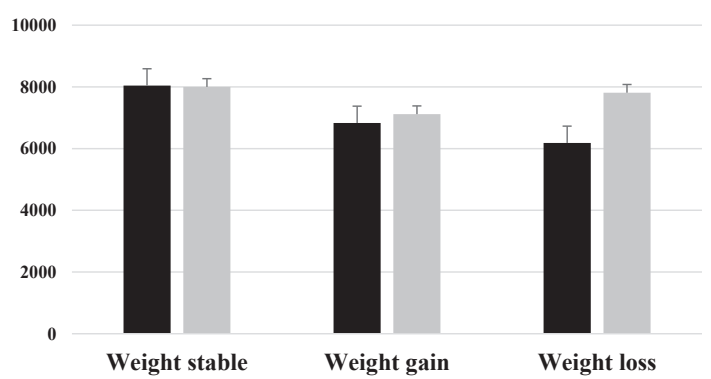

Figure 2 AUC responses of glucose, insulin, GLP-1 and GIP after OGTT by weight group. Weight stable $\mathrm{n}=30$, weight gain $\mathrm{n}=27$, weight loss $\mathrm{n}=9$. Data are mean \pm SEM. The given $\mathrm{p}$-value shows differences between before and after fructose measurement, all other variables did not change significantly (Wilcoxon signed rank test).

\section{Discussion}

This is the first study to report the effect of fructose consumption in obese non-diabetic males on incretin responses following both OGTT and mixed meal. We show

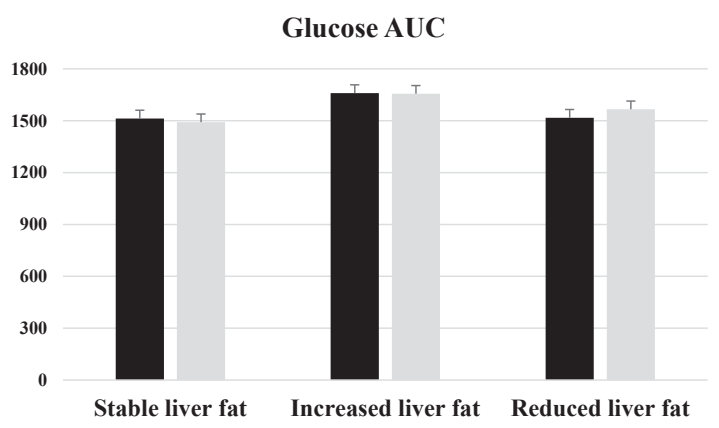

GLP-1 AUC

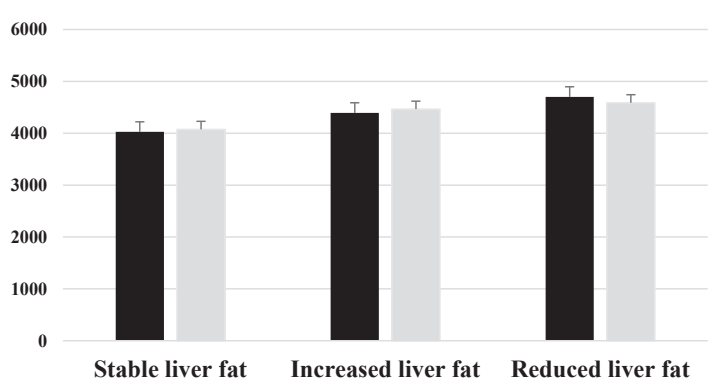

that despite mild but significant weight and liver fat gain accompanied with fasting and postprandial TG increase, fructose consumption had no impact on either glycemic control or GLP-1 and GIP responses. Furthermore, although subgroups who gained either weight or liver fat had worse

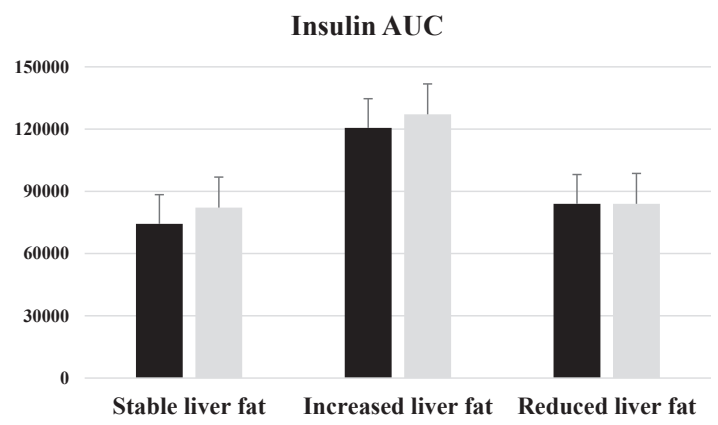

GIP AUC

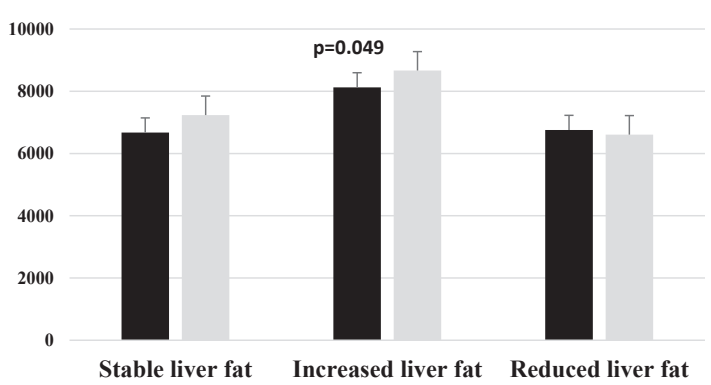

Figure 3 AUC responses of glucose, insulin, GLP-1 and GIP after OGTT by liver fat group. Stable liver fat $\mathrm{n}=17$, increased liver fat $\mathrm{n}=27$, reduced liver fat $\mathrm{n}=22$. Data are mean \pm SEM. The given $\mathrm{p}$-value shows differences between before and after fructose measurement, all other variables did not change significantly (Wilcoxon signed rank test). 
TG profiles, this was not associated with worsening of HOMA-IR, glucose tolerance during 4 h OGTT or GLP-1 or GIP responses measured either during OGTT or mixed meal. As expected, the energy intake was slightly hypercaloric during fructose intervention. However actual change of caloric intake was not significant as the subjects adjusted their caloric intake from other food and drinks and also fasting and postprandial PYY concentrations reflecting satiety remained stable. So our results suggest that daily fructose intake of $75 \mathrm{~g}$ consumed with a close to weight-maintaining diet does not in itself alter glycemia, but has adverse effects on lipid metabolism.

Our study population was obese, with a large waist circumference, and $41 \%$ had elevated screening TG concentrations, and as such, consisted of a high diabetes risk population that should be more prone to develop acquired insulin resistance. The present findings are in contradiction with strong, positive epidemiologic associations reported between fructose intake and components of the metabolic syndrome, insulin resistance and diabetes $[1,25]$. On the other hand, clinical trials have not been as consistent. Although fructose consumption increases liver fat, fasting TG, and systolic blood pressure and decreases HDL [26], not all studies report impaired glycemic variables. A recent meta-analysis of 29 published studies described effects of fructose in 1005 normal-weight and overweight or obese non-diabetic participants, and concluded that isocaloric or hypercaloric fructose impairs hepatic insulin sensitivity but not peripheral or muscle insulin sensitivity [27]. The included studies were shorter than 8 weeks except for 3 studies, had lower numbers of participants, except for 2 studies, and mostly measured only fasting glucose and insulin. Our results from a 12 weeks fructose intervention in a rather large study population are in agreement with the results from the metaanalysis regarding fasting insulin and HOMA-IR, and extend the findings to glycemic control and incretin responses in $4 \mathrm{~h}$ OGTT.

The concentration curves for glucose, insulin, GLP-1 and GIP before and after the intervention are almost superimposable (Fig. 1), and consistent with a stable glycemia also confirmed by calculation of Matsuda and Insulinogenic indexes. Whether previous findings of deleterious effects of fructose on insulin sensitivity are due to direct metabolic effects of fructose itself or a result of increased energy intake and weight gain remains unanswered. We have previously reported a defective GLP-1 response in acquired obesity with liver fat accumulation in young twins [16]. In contrast, in our current study population, the overall weight increase was minor, less than $1 \mathrm{~kg}$, but even when the data were analyzed according to the weight change during the intervention, we did not find that weight gain was associated with impaired glycemic control or incretin responses. Thus, results from studies conducted in free-living conditions may be modulated by metabolic adaptions to nutrient and caloric intake [28,29].

We observed a range of liver fat changes during the fructose intervention, although the average absolute increase was only 0.6 percentage points, from $6.9 \%$ to $7.5 \%$ but a clear increase in postprandial TG AUC and AUIC. Therefore, we analyzed more closely if liver fat gain during fructose is a key determinant of abnormal glucose and lipid metabolism. However, when data were analyzed according to changes in liver fat, even the group with the greatest increase (fat from $8.7 \%$ to $11.3 \%$ ) had no changes in glucose, insulin or GLP-1 responses during OGTT, with only marginally significant increases in GIP AUC despite the worse cardiometabolic profile. The postprandial incretin responses after the mixed meal were also not related to changes in liver fat, although the group with increased liver fat did have a significant increase in TG AUC response. This may indicate a disconnect between pathways of lipid and glucose metabolism. The concept of pathway-selective insulin resistance and responsiveness [30] is consistent with the results in our population.

We have previously shown that in obese males, GLP-1, GLP-2 and GIP responses to a mixed meal test are greater than following an OGTT. However, the contribution of these hormones to the variance in postprandial TG was minor [31]. This also is in concordance with our present results showing that incretin responses do not correlate significantly to impairment of lipid metabolism in obese nondiabetic males and that fructose intervention leading to increased TG response does not have any modulating effect on gut incretins during a mixed meal. We conclude that in these subjects, fructose consumption has little effect on the gut incretin-pancreas pathway or glucose tolerance.

Our study has some strengths and limitations. The time frame of 12 weeks is longer than in earlier acute or shortterm mechanistic studies [27]. A longer intervention trial assessing insulin sensitivity with simple measurements in young adults [32] did not find differences between the effects of glucose and fructose. With respect to the findings that changes in the incretin system are early signs of altered glucose and insulin [15,16], we assume that changes in gut-pancreas axis would be detectable at the same time when changes in lipid metabolism are seen. Taken into account that our intervention period is substantially shorter than follow-up in previous epidemiologic studies that demonstrate impaired glucose tolerance and risk for diabetes with fructose consumption, our results may not be extrapolated to habitual and life-long use of excess fructose. Other limitations include lack of measurement of incretin-induced insulin secretion, lack of control group and standardized background diet, use liquid fructose beverage rather than naturally occurring sucrose and no measure of the absorption of fructose. However, our in our study the amount of fructose and the way it was served were chosen to mimic a behavior which is prevalent in Western societies [29].

In summary, our data show that $75 \mathrm{~g}$ daily fructose from SSBs for 12 weeks does not impair gut incretin response or glucose tolerance in obese males, even in the presence of moderate weight- or liver fat gain. Based on our results, the impairment of hepatic lipid handling and dyslipidemia in non-diabetic obese men due to fructose consumption may represent an early metabolic dysfunction in the metabolism of fructose. Our results 
show that these early adverse changes are not linked to worsening of glucose tolerance or dysregulation of gut incretins in subjects in high risk for diabetes, at least within the dose and time frame studied here.

\section{Sources of funding}

This paper was supported by grants from Helsinki University Central Hospital EVO-funds, Academy of Finland (grants 266286 and 272376), European Foundation for the Study of Diabetes, Sigrid Juselius Foundation, Yrjö Jahnsson Foundation, Juho Vainio Foundation, Foundation Leducq France, Swedish Research Council, Swedish Heart Lung Foundation, Diabetes Research Wellness Foundation, Sahlgrenska University Hospital ALF grant, Swedish Diabetes Foundation, the NovoNordisk Foundation, and the EU Project RESOLVE.

\section{Disclosures}

M.R.T. from Sanofi-Aventis, Lilly, Merck Sharp \& Dohme, Kowa, Novartis, Novo Nordisk, and AstraZeneca; J.B. from AstraZeneca, Sanofi-Aventis and Merck Sharp \& Dome; C.F.D. from Boehringer Ingelheim, Lilly, MSD and Novo Nordisk; K.H.P. from Merck/MSD, Novo Nordisk, and AstraZeneca; and B.E. from Novo Nordisk, Sanofi, Eli Lilly, MSD and Boehringer Ingelheim.

\section{Conflict of interest}

None.

\section{Acknowledgments}

We are grateful to Hannele Hilden, Helinä Perttunen-Nio, Elisa Koivisto, Sylvain Pouliot, Elin Stenfeldt, Eva HedmanSabler and Sofie Pilgaard for excellent laboratory work and patient care.

\section{Appendix A. Supplementary data}

Supplementary data related to this article can be found at http://dx.doi.org/10.1016/j.numecd.2017.03.003.

\section{References}

[1] Malik VS, Hu FB. Fructose and cardiometabolic health: what the evidence from sugar-sweetened beverages tells us. J Am Coll Cardiol 2015;66(14):1615-24.

[2] Bray GA. How bad is fructose? Am J Clin Nutr 2007;86(4):895-6.

[3] Chung M, Ma J, Patel K, Berger S, Lau J, Lichtenstein AH. Fructose, high-fructose corn syrup, sucrose, and nonalcoholic fatty liver disease or indexes of liver health: a systematic review and metaanalysis. Am J Clin Nutr 2014;100(3):833-49.

[4] Sievenpiper JL, de Souza RJ, Cozma AI, Chiavaroli L, Ha V, Mirrahimi A. Fructose vs. glucose and metabolism: do the metabolic differences matter? Curr Opin Lipidol 2014;25(1):8-19.

[5] Stanhope KL, Medici V, Bremer AA, Lee V, Lam HD, Nunez MV, et al. A dose-response study of consuming high-fructose corn syrup-sweetened beverages on lipid/lipoprotein risk factors for cardiovascular disease in young adults. Am J Clin Nutr 2015; 101(6):1144-54.

[6] Johnston RD, Stephenson MC, Crossland H, Cordon SM, Palcidi E Cox EF, et al. No difference between high-fructose and highglucose diets on liver triacylglycerol or biochemistry in healthy overweight men. Gastroenterology 2013;145(5):1016-25. e2.

[7] Ngo Sock ET, Le KA, Ith M, Kreis R, Boesch C, Tappy L. Effects of a short-term overfeeding with fructose or glucose in healthy young males. Br J Nutr 2010;103(7):939-43.

[8] Silbernagel G, Machann J, Unmuth S, Schick F, Stefan N, Häring HU, et al. Effects of 4-week very-high-fructose/glucose diets on insulin sensitivity, visceral fat and intrahepatic lipids: an exploratory trial. Br J Nutr 2011;106(1):79-86.

[9] Maersk M, Belza A, Stodkilde-Jorgensen H, Ringgaard S, Chabanova E, Thomsen $\mathrm{H}$, et al. Sucrose-sweetened beverages increase fat storage in the liver, muscle, and visceral fat depot: a 6-mo randomized intervention study. Am J Clin Nutr 2012;95(2):283-9.

[10] Bravo S, Lowndes J, Sinnett S, Yu Z, Rippe J. Consumption of sucrose and high-fructose corn syrup does not increase liver fat or ectopic fat deposition in muscles. Appl Physiol Nutr Metab 2013; 38(6):681-8.

[11] Lecoultre V, Egli L, Carrel G, Theytaz F, Kreis R, Schneiter P, et al. Effects of fructose and glucose overfeeding on hepatic insulin sensitivity and intrahepatic lipids in healthy humans. Obesity (Silver Spring) 2013;21(4):782-5.

[12] Stanhope KL, Schwarz JM, Keim NL, Griffen SC, Bremer AA, Graham JL, et al. Consuming fructose-sweetened, not glucosesweetened, beverages increases visceral adiposity and lipids and decreases insulin sensitivity in overweight/obese humans. J Clin Investig 2009;119(5):1322-34.

[13] Aeberli I, Hochuli M, Gerber PA, Sze L, Murer SB, Tappy L, et al. Moderate amounts of fructose consumption impair insulin sensitivity in healthy young men: a randomized controlled trial. Diabetes Care 2013;36(1):150-6.

[14] Meier JJ. GLP-1 receptor agonists for individualized treatment of type 2 diabetes mellitus. Nat Rev Endocrinol 2012;8(12):728-42.

[15] Vaag AA, Holst JJ, Volund A, Beck-Nielsen HB. Gut incretin hormones in identical twins discordant for non-insulin-dependent diabetes mellitus (NIDDM)-evidence for decreased glucagon-like peptide 1 secretion during oral glucose ingestion in NIDDM twins. Eur J Endocrinol 1996;135(4):425-32.

[16] Matikainen N, Bogl LH, Hakkarainen A, Lundbom J, Lundbom N, Kaprio J, et al. GLP-1 responses are heritable and blunted in acquired obesity with high liver fat and insulin resistance. Diabetes Care 2014;37(1):242-51.

[17] Kuhre RE, Gribble FM, Hartmann B, Reimann F, Windeløv JA Rehfeld JF, et al. Fructose stimulates GLP-1 but not GIP secretion in mice, rats, and humans. Am J Physiol Gastrointest Liver Physiol 2014;306(7):G622-30.

[18] Wolnerhanssen BK, Meyer-Gerspach AC, Schmidt A, Zimak N, Peterli R, Beglinger C, et al. Dissociable behavioral, physiological and neural effects of acute glucose and fructose ingestion: a pilot study. PLoS One 2015;10(6):e0130280.

[19] Vozzo R, Baker B, Wittert GA, Wishart JM, Morris H, Horowitz M, et al. Glycemic, hormone, and appetite responses to monosaccharide ingestion in patients with type 2 diabetes. Metabolism 2002;51(8):949-57.

[20] Lundbom J, Hakkarainen A, Soderlund S, Westerbacka J, Lundbom N, Taskinen MR. Long-TE 1HMRS suggests that liver fat is more saturated than subcutaneous and visceral fat. NMR Biomed $2011 ; 24(3): 238-45$.

[21] Deacon CF, Holst JJ. Immunoassays for the incretin hormones GIP and GLP-1. Best Pract Res Clin Endocrinol Metab 2009;23(4): 425-32.

[22] Lindgren O, Carr RD, Deacon CF, Holst JJ, Pacini G, Mari A, et al. Incretin hormone and insulin responses to oral versus intravenous lipid administration in humans. J Clin Endocrinol Metab 2011; 96(8):2519-24.

[23] Orskov C, Rabenhoj L, Wettergren A, Kofod H, Holst JJ. Tissue and plasma concentrations of amidated and glycine-extended glucagon-like peptide I in humans. Diabetes 1994;43(4):535-9.

[24] Matthews DR, Hosker JP, Rudenski AS, Naylor BA, Treacher DF, Turner RC. Homeostasis model assessment: insulin resistance and beta-cell function from fasting plasma glucose and insulin concentrations in man. Diabetologia 1985;28(7):412-9. 
[25] Romaguera D, Norat T, Wark PA, Vergnaud AC, Schulze MB, van Woudenbergh GJ, et al., for the InterAct Consortium. Consumption of sweet beverages and type 2 diabetes incidence in European adults: results from EPIC-InterAct. Diabetologia 2013;56:1520-30.

[26] Kelishadi R, Mansourian M, Heidari-Beni M. Association of fructose consumption and components of metabolic syndrome in human studies: a systematic review and meta-analysis. Nutrition 2014;30:503-10.

[27] ter Horst KW, Schene MR, Holman R, Romijn JA, Serlie MJ. Effect of fructose consumption on insulin sensitivity in nondiabetic subjects: a systematic review and meta-analysis of diet-intervention trials. Am J Clin Nutr 2016;104(6):1562-76.

[28] Augustin LS, Kendall CW, Jenkins DJ, Willett WC, Astrup A, Barclay AW, et al. Glycemic index, glycemic load and glycemic response: an International Scientific Consensus Summit from the
International Carbohydrate Quality Consortium (ICQC). Nutr Metab Cardiovasc Dis 2015;25(9):795-815.

[29] Laughlin MR, Bantle JP, Havel PJ, Parks E, Klurfeld DM, Teff K, et al. Clinical research strategies for fructose metabolism. Adv Nutr 2014;5:248-59.

[30] Williams KJ, Wu X. Imbalanced insulin action in chronic over nutrition: clinical harm, molecular mechanisms, and a way forward. Atherosclerosis 2016;247:225-82.

[31] Matikainen N, Bjornson E, Soderlund S, Borén C, Eliasson B, Pietiläinen KH, et al. Minor contribution of endogenous GLP-1 and GLP-2 to postprandial lipemia in obese men. PLoS One 2016;11(1): e0145890.

[32] Huttunen JK, Mäkinen KK, Scheinin A. Turku sugar studies XI Effects of sucrose, fructose and xylitol diets on glucose, lipid and urate metabolism. Acta Odontol Scand 1976;34(6):345-51. 